\title{
BMJ Open Randomised, double-blind, placebo- controlled clinical trial investigating the effects of inorganic nitrate in hypertension-induced target organ damage: protocol of the NITRATE-TOD study in the UK
}

Clement Wai Zhen Lau (D) ,,2 Alexander Jozua Pedro Hamers, ${ }^{1}$ Krishnaraj Sinhji Rathod, ${ }^{1,2}$ Asad Shabbir, ${ }^{1}$ Jackie Cooper, ${ }^{1}$ Christopher Peter Primus, ${ }^{1,2}$ Ceri Davies, ${ }^{1,2}$ Anthony Mathur, ${ }^{1,2}$ James C Moon, ${ }^{2,3}$ Vikas Kapil, ${ }^{1,2}$ Amrita Ahluwalia ${ }^{1}$

To cite: Lau CWZ, Hamers AJP, Rathod KS, et al. Randomised, double-blind, placebo-controlled clinical trial investigating the effects of inorganic nitrate in hypertension-induced target organ damage: protocol of the NITRATE-TOD study in the UK. BMJ Open 2020;10:e034399. doi:10.1136/ bmjopen-2019-034399

- Prepublication history for this paper is available online. To view these files, please visit the journal online (http://dx.doi. org/10.1136/bmjopen-2019034399).

Received 19 September 2019 Revised 20 November 2019 Accepted 22 November 2019

Check for updates

(C) Author(s) (or their employer(s)) 2020. Re-use permitted under CC BY-NC. No commercial re-use. See rights and permissions. Published by BMJ.

For numbered affiliations see end of article.

Correspondence to Professor Amrita Ahluwalia; a.ahluwalia@qmul.ac.uk

\section{ABSTRACT}

Introduction Arterial stiffness and left ventricular (LV) hypertrophy are the key markers of hypertensive target organ damage (TOD) associated with increased cardiovascular morbidity and mortality. We have previously shown that dietary inorganic nitrate supplementation lowers blood pressure (BP) in hypertension, however, whether this approach might also improve markers of hypertensive TOD is unknown. In this study, we will investigate whether daily dietary inorganic nitrate administration reduces LV mass and improves measures of arterial stiffness.

Methods and design NITRATE-TOD is a double-blind, randomised, single-centre, placebo-controlled phase II trial aiming to enrol 160 patients with suboptimal BP control on one or more antihypertensives. Patients will be randomised to receive 4 months once daily dose of either nitrate-rich beetroot juice or nitrate-deplete beetroot juice (placebo). The primary outcomes are reduction in LV mass and reduction in pulse wave velocity (PWV) and central BP.

The study has a power of $95 \%$ for detecting a $9 \mathrm{~g} \mathrm{LV}$ mass change by cardiovascular MRI ( 6\% change for a mildly hypertrophied heart of $150 \mathrm{~g}$ ). For PWV, we have a power of $>95 \%$ for detecting a $0.6 \mathrm{~m} / \mathrm{s}$ absolute change. For central systolic BP, we have a $>90 \%$ power to detect a $5.8 \mathrm{~mm} \mathrm{Hg}$ difference in central systolic BP.

Secondary end points include change in ultrasound flowmediated dilation, change in plasma nitrate and nitrite concentration and change in BP.

Ethics and dissemination The study was approved by the London-City and East Research Ethics Committee (10/H0703/98). Trial results will be published according to the Consolidated Standards of Reporting Trials statement and will be presented at conferences and reported in peerreviewed journals.

Trial registration number NCT03088514
Strengths and limitations of this study

- This is a randomised, placebo-controlled doubleblind clinical trial assessing the potential of dietary nitrate in reducing both cardiac and vascular damage due to persistently elevated blood pressure.

- Dietary nitrate is a simple, easy to use and safe intervention that may be more acceptable to patients on long-term medication for their blood pressure.

- This is a single-centre phase II study; therefore, the applicability to other centres is uncertain.

\section{INTRODUCTION}

Hypertension is the leading risk factor for cardiovascular diseases (CVDs) worldwide. $^{12}$ In 2016, an estimated 17.9 million people died from CVDs, which represents $31 \%$ of all global deaths. ${ }^{3}$ Approximately half of these deaths were caused by complications from hypertension. ${ }^{4}$ Hence, novel and costeffective therapeutic strategies continue to be sought. In this regard, there has been a major emphasis to increase vegetable intake since increased consumption of a vegetablerich diet particularly green leafy vegetables confers protection against CVD, including the lowering of blood pressure (BP). ${ }^{56}$ One particular constituent of such vegetables that has been proposed to underlie the beneficial effects of this food group is inorganic nitrate; through bioconversion to nitrite, and then nitrite to nitric oxide $(\mathrm{NO})^{7}$ in the body. Importantly, provision of inorganic nitrate to 
hypertensive patients causes a rise in plasma nitrate and is associated with a decrease in $\mathrm{BP}^{89}$

Dietary inorganic nitrate once ingested rapidly enters the circulation ${ }^{8-10}$ with a proportion of this nitrate being extracted and then secreted and concentrated in the saliva. ${ }^{11}$ Bacteria residing in the oral cavity convert this nitrate to nitrite which is then swallowed ${ }^{12}$; appearing soon after within the circulation. We have recently shown that daily supplementation with dietary nitrate (providing approximately $6.4 \mathrm{mmol}$ nitrate daily) for 4 weeks was associated with robust, sustained and clinically meaningful reductions in BP (measured by clinic, ambulatory and home methods) of $\sim 8 / 4 \mathrm{~mm} \mathrm{Hg} .{ }^{13}$ However, persistent hypertension leads to both vascular and cardiac remodelling and whether inorganic nitrate-induced reductions in BP might be associated with improvements in either of these in the long term is unknown. Experimentally in patients one can assess this potential through the measurement of arterial stiffness and left ventricular (LV) mass, respectively, with increases of either conferring increased CV risk. Arterial stiffness (and its inverse measure, distensibility) refers to the decreased elasticity that develops in arteries as a consequence of vascular remodelling resulting from alterations of the fibrous components of the extracellular matrix (including elastin and collagen) ${ }^{14}$ Importantly, increased arterial stiffness, as measured using carotid-femoral pulse wave velocity $(\mathrm{PWV}),{ }^{15}$ is a strong predictor of CV events, ${ }^{16}$ and there have been suggestions that drug development targeting this phenomenon is likely to provide a new genre of therapeutics in the combat against CVD. ${ }^{17}$ Additionally, an increased central pulse pressure occurs when the large conduit blood vessels lose their elasticity and become less able to accommodate all of the blood ejected from the heart. This increase in pressure results in an increase in central systolic BP and causes an increase in the stress imposed on the left ventricle, which in turn can result in LV hypertrophy (LVH). ${ }^{18}$ Elevated LV mass is an initial compensatory mechanism to normalise wall stress due to elevated BP. However, this cardiac remodelling eventually becomes maladaptive with an increase in myocardial oxygen consumption, cardiac chamber dilation, reduced LV contractility and progressive deterioration to heart failure. ${ }^{19}$ The measure of $\mathrm{LVH}$ as a prognostic indicator of adverse outcome was first convincingly identified in the Framingham study, ${ }^{20}$ and numerous studies since have confirmed the strong relationship between LVH and CV events and mortality. ${ }^{21}{ }^{22}$ Perhaps, more importantly, LV mass reduction is associated with further improvements in $\mathrm{CV}$ outcomes. ${ }^{23} 24$

It has been proposed that insufficient supply of endogenous NO likely contributes to the progression and worsening of arterial stiffness and LVH. ${ }^{25}$ Preclinical studies have demonstrated that an elevation of systemic nitrite levels reduces cardiac hypertrophy in mice. ${ }^{26}$ Moreover, administration of inorganic nitrate reduces PWV healthy volunteers and hypertensive patients in shortterm studies. ${ }^{1327}$ It is also possible that decreasing arterial stiffness improves ventricular-arterial coupling which might result in benefits in cardiac function. ${ }^{28}$

The objective of this study is to determine whether a dietary nitrate intervention might elevate the levels of NO in the body sufficiently to alter both heart and blood vessel remodelling in hypertensive patients with suboptimal BP control.

\section{METHODOLOGY}

Trial objectives

Aims of research

We wish to test the hypothesis that dietary supplementation of inorganic nitrate (in beetroot juice) elevates circulating nitrate and nitrite levels ultimately delivering NO to the vasculature and thereby improving endothelial, vascular and cardiac function in patients with treated yet uncontrolled hypertension. Specifically, the aim is to investigate whether prolonged dietary nitrate ingestion in hypertensive patients with suboptimal BP control, can cause reductions in LV mass and PWV.

\section{Participant selection}

This is a single-centre study, in which patients will be identified and recruited at Barts Health National Health Service (NHS) Trust. In addition, other participant identification centres will be used to identify suitable participants via the National Institute for Health Research (NIHR) Clinical Research Network and local NHS Trusts.

\section{Original hypothesis}

Dietary inorganic nitrate ingestion, in addition to existing pharmacological therapy, reduces BP leading to reductions in LV mass and arterial stiffness.

\section{Primary end points}

The primary end points are change in LV mass, determined using cardiovascular MRI (CMR) (NITRATE-LVH arm) and a change in central systolic BP and PWV (NITRATE-CBP arm).

\section{Secondary end points}

Secondary endpoints are change in ultrasound determined brachial artery flow-mediated dilation (FMD), change in plasma nitrate and nitrite levels and a change in peripheral BP.

\section{Exploratory end points}

Exploratory end points for cardiac imaging include a change in aortic distensibility, LV systolic and diastolic function, LV volumes and ejection fraction, left atrial volumes and function and markers of myocardial fibrosis and oedema. We will assess changes in 12-lead ECG parameters of LVH. With regard to BP, we will assess for changes in $\mathrm{BP}$ variability, $\mathrm{BP}$ control rate and $\mathrm{BP}$ circadian pattern. We will also perform analysis of the salivary microbiome. Finally, we will assess changes in quality of life score (EQ-5D questionnaire) and biochemical 
measures for example, urine albumin excretion, cholesterol fractions, B-natriuretic peptide and troponin $\mathrm{T}$.

\section{INCLUSION CRITERIA}

A subject will be eligible for inclusion in this study only if all of the following criteria apply:

1. Patients will be enrolled following an informed consent.

2. Aged $18-80$ years.

3. The study subjects will be hypertensive with evidence of difficulty treating to target $\mathrm{BP}$ (daytime ambulatory BP monitor 135-170 and/or 85-105 mm Hg) on one or more antihypertensive agent, with insufficient efficacy or intolerance of medications.

4. For the NITRATE-LVH arm, echocardiographic evidence of LVH (LV mass indexed to body surface area (BSA); males $>115 \mathrm{~g} / \mathrm{m}^{2}$; females $>95 \mathrm{~g} / \mathrm{m}^{2}$ ).

5. Patients will have been established on an antihypertensive treatment regime for at least 1 month by the time of participation in the study and will not require changes in pharmacological intervention for the duration of the trial.

\section{EXCLUSION CRITERIA}

Unless specified, a subject will not be eligible for inclusion in this study if any of the following criteria apply:

1. History of chronic viral hepatitis (including presence of hepatitis B surface antigen or hepatitis C antibody) or other chronic hepatic disorders.

2. History of increased liver function tests (alanine transaminase (ALT), aspartate transaminase (AST)) due to acute or chronic liver conditions, $3 \mathrm{x}$ above the upper limit of normal or bilirubin 1.5x above the upper limit of normal at screening.

3. Renal impairment with creatinine clearance (estimated glomerular filtration rate (eGFR)) of $<50 \mathrm{~mL} / \mathrm{min}$ at screening.

4. Patients with diabetes mellitus defined by previous history of diabetes or haemoglobin Alc (HbAlc) $>6.5 \%(>48 \mathrm{mmol} / \mathrm{mol})$ at screening.

5. Subjects with low-density lipoprotein cholesterol (LDLc), $>7.5 \mathrm{mmol} / \mathrm{L}$ and/or triglyceride level $>10 \mathrm{mmol} / \mathrm{L}$.

6. History of heart failure defined as New York Heart Association (NYHA) class II-IV or those with known LV dysfunction (LV ejection fraction $<40 \%$ ) regardless of symptomatic status.

7. History of malignancy within the past 5 years, other than non-melanoma skin cancer.

8. Current life-threatening condition other than vascular disease (eg, very severe chronic airways disease, HIV positive, life-threatening arrhythmias) that may prevent a subject from completing the study.

9. Use of an investigational device or investigational drug within 30 days or five half-lives (whichever is longer) preceding the first dose of study medication.
10. Subjects who will commence or who are likely to commence regular treatment with non-steroidal anti-inflammatory drugs (other than aspirin), from screening until study completion.

11. Any non-stable dosing of ongoing medication regimens throughout the study trial.

12. Drug abuse within the past 6 months.

13. The subject has a 3-month prior history of regular alcohol consumption exceeding an average weekly intake of $>28$ units (or an average daily intake of greater than three units) for males, or an average weekly intake of $>21$ units (or an average daily intake of greater than two units) for females. 1 unit is equivalent to a half-pint $(284 \mathrm{~mL})$ of beer/lager; $25 \mathrm{~mL}$ measure of spirits or $125 \mathrm{~mL}$ of wine.

14. Any other subject whom the investigator deems unsuitable for the study (eg, due to other medical reasons, laboratory abnormalities, expected study medication non-compliance or subject's unwillingness to comply with all study-related study procedures).

15. Subjects with rheumatoid arthritis, connective tissue disorders and other conditions known to be associated with chronic inflammation (eg, inflammatory bowel disease).

16. Subjects with any acute infection or recent systemic (oral or intravenous) antibiotics within 1 month of screening, or significant trauma (burns, fractures).

17. Subjects who have donated more than $500 \mathrm{~mL}$ of blood within 56 days prior to the study medication administration.

18. Self-reported use of antimicrobial mouthwash or tongue scrapes.

19. Concomitant xanthine oxidase inhibitors (such as allopurinol).

20. Known history of significant claustrophobia, previous intolerance of CMR or known (or suspected) incompatible metallic implant.

21. Pregnancy.

22. Allergy to gadolinium-based contrast agents used for CMR.

23. Patients with known LVH caused by another established pathology diagnosed prior to or at screening, for example, severe aortic stenosis, hypertrophic cardiomyopathy, amyloidosis and Fabry disease.

Exceptions to the exclusion criteria:

- For criteria 18, patients can enter the trial if they discontinue the use of antimicrobial mouthwash for the duration of the clinical trial.

- Criteria 20 and 22 do not apply to participants who will not have a CMR scan in the NITRATE-CBP arm.

\section{Study design and intervention}

This is a prospective double-blind, placebo-controlled, clinical study. A total of 160 patients (male and female, age 18-80) with hypertension as per requirements indicated above will be recruited. Figure 1 shows a summary of the study scheme. Patients will be stratified at screening into two arms. Participants with LVH at screening 


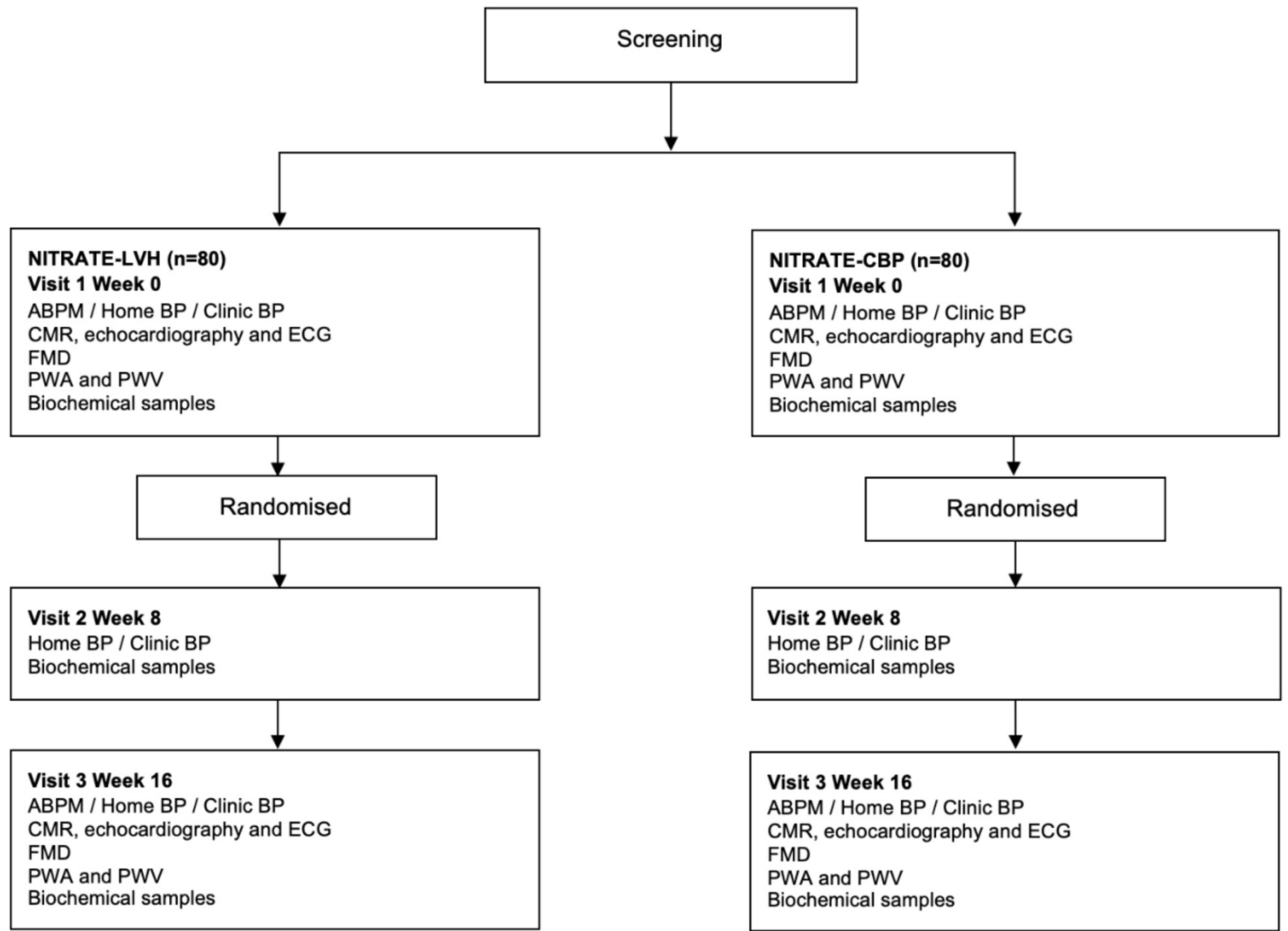

Figure 1 Study protocol. ABPM, ambulatory blood pressure monitor; BP, blood pressure; CBP, central blood pressure; CMR, cardiovascular magnetic resonance; FMD, flow-mediated dilation; LVH, left ventricular hypertrophy; PWA, pulse wave analysis; PWV, pulse wave velocity.

echocardiography will enter the NITRATE-LVH arm $(\mathrm{n}=80)$ while those who do not have LVH but satisfy the other eligibility criteria will enter the NITRATE-CBP arm $(\mathrm{n}=80)$. LVH is defined by increased LV mass determined by acquiring a parasternal long-axis image and subsequently measured for the interventricular septal wall thickness, end-diastolic LV cavity diameter and posterior $\mathrm{LV}$ wall thickness according to published guidelines. ${ }^{29} \mathrm{LV}$ mass is calculated as previously described and indexed to BSA. $^{30}$

All visits will take place at the William Harvey Heart Centre, Queen Mary University of London and Barts Heart Centre, St Bartholomew's Hospital, Barts Health NHS Trust. Patients will be randomised to receive $70 \mathrm{~mL}$ of a beetroot juice concentrate containing $\sim 6 \mathrm{mmol}$ nitrate or nitrate-depleted placebo juice concentrate (James White Drinks, UK) control. The volunteers will start taking their daily dose after completion of their baseline visit (visit 1) for 16 weeks. Patients will be advised to take their dose of juice at the same time each day, preferably in the morning with their breakfast. Patients will be provided with dietary advice in relation to the calorific content of interventions (both $\sim 100 \mathrm{kcal}$ ). In addition to baseline and 16 week visits, patients will return to the clinic at 8 weeks for collection of samples for NOx analysis and for discussion of any adherence issues.

\section{Randomisation and blinding process}

Patients will be block randomised on a 1:1 basis to receive either dietary nitrate or placebo, using a binary random number sequence (http://www.random.org). Treatment assignment for volunteers in the dietary nitrate and placebo groups will remain blinded until data lock and statistical analysis at the end of the study. If unblinding is required, the chief investigator for the study will be informed. A list of the unblinded treatments will be kept in a secure location at the Barts Cardiovascular Clinical Trials Unit (CVCTU). The unblinding procedure will be available at all times.

\section{Patient and public involvement}

Patients were directly involved in the design and planning of the study. Twenty previous Barts Health NHS Trust 
patients were surveyed regarding aspects of trials involving dietary interventions, which informed this protocol.

\section{Study start and end dates}

Recruitment commenced on 23 March 2017. The provisional end date for recruitment is September 2020.

\section{Methods to be used}

Blood, saliva and urine analysis

In this study, venous blood will be taken using a 21-gauge butterfly needle at each visit. Blood samples will be centrifuged immediately and plasma separated. The plasma samples will be snap frozen in liquid nitrogen and stored at $-80^{\circ} \mathrm{C}$. These samples will be used for the purposes of making biochemical measurements and will be discarded once used. Similarly, saliva and urine will be collected at each visit. Saliva will be centrifuged, and a pellet generated. This pellet contains oral bacteria that have dislodged from the oral cavity. This pellet will be frozen for later analysis of oral bacteria by second-generation genome sequencing.

Nitrate and nitrite levels in saliva, blood and urine samples will be determined using ozone chemiluminescence as previously described. ${ }^{31}{ }^{32}$ In brief, total nitrate and nitrite concentration (termed 'NOx') will be determined by adding samples to $0.1 \mathrm{~mol} / \mathrm{L}$ vanadium (III) chloride in $1 \mathrm{M}$ hydrochloric acid refluxing at $95^{\circ} \mathrm{C}$ under nitrogen. Nitrite concentration will be determined by the addition of samples to $0.09 \mathrm{~mol} / \mathrm{L}$ potassium iodide in glacial acetic acid under nitrogen at room temperature. Nitrate concentration will be calculated by the subtraction of (nitrite) from (NOx). All measurements will be conducted by an individual blinded to the intervention. In addition, a sample from each batch of juice sent to each volunteer will be analysed for NOx content.

\section{Pulse wave analysis and pulse wave velocity}

Pulse wave analysis (PWA) and PWV are the measures of arterial stiffness, which will be determined by a noninvasive Vicorder device (Skidmore Medical, Bristol, UK). For PWA, the pulse wave will be recorded from the brachial cuff applied to the non-dominant arm. For PWV, the pulse wave will be simultaneously recorded from the carotid and femoral site using an oscillometric method. A small, inflatable neck pad will be placed directly over a carotid artery and secured around the neck by a Velcro tab and a cuff will also be placed around the patient's ipsilateral upper thigh. Both carotid and femoral cuffs will be simultaneously inflated automatically to $65 \mathrm{~mm} \mathrm{Hg}$ and the corresponding oscillometric signal from each cuff digitally analysed to extract the pulse time delay. To estimate the aortic length, the distance between the sternal notch and the thigh cuff will be measured. From these measurements, PWV can be derived as PWV=aortic distance/pulse time delay. ${ }^{33}$

\section{Flow-mediated dilation}

FMD will be used to non-invasively assess endothelial function, using vascular ultrasound to measure the increase in brachial artery diameter in response to increased flow ${ }^{34}$ as previously described. ${ }^{35} \mathrm{~A}$ high-resolution external vascular ultrasound Siemens/Acuson Sequoia C256 Colour Doppler with a $7.0 \mathrm{MHz}$ linear-array transducer supported by a stereotactic clamp will be used to assess the vessel diameter in the right arm. The vessel will be scanned in longitudinal section and the centre will be identified when the clearest views of the anterior and posterior artery walls have been obtained. Images will be magnified with a resolution box function and images of the brachial artery acquired continuously using semiautomated edge detection software (FMD Studio, Quipu s.r.l, Pisa, Italy) and analysed in real time. An automatic mathematical contour tracking operator locates the pulsed-wave Doppler of the brachial artery, which will be used to measure the diameter and blood flow velocity continuously for $1 \mathrm{~min}$ at baseline, then during $5 \mathrm{~min}$ of reduced blood flow (induced by inflation to $300 \mathrm{~mm} \mathrm{Hg}$ of a pneumatic cuff placed on the right forearm site distal to the segment of artery being analysed), and finally for a further 5 min during the reactive hyperaemia following cuff release. FMD is defined as the maximum percentage increase in vessel diameter during the reactive hyperaemia. This procedure will be performed at visit 1 (baseline) and visit 3 (16 weeks). In some volunteers, following FMD, $0.4 \mathrm{mg}$ of sublingual glyceryl trinitrate will be administered at visit 1 and visit 3 to determine whether changes in FMD responses following intervention might be due to changes in smooth muscle reactivity.

\section{Transthoracic echocardiography}

At visits 1 and 3, echocardiography is also performed to assess LV diastolic function. Images will be acquired for measures of LV diastolic function including left atrial end-diastolic volumes, pulmonary vein $\mathrm{s} / \mathrm{d}$ ratio, mitral valve $\mathrm{E} / \mathrm{A}$ ratio and $\mathrm{E} / \mathrm{e}^{\prime} \cdot{ }^{29}{ }^{36}$ Images will be acquired and analysed by an operator accredited by the British Society of Echocardiography.

\section{Cardiovascular MRI}

The most accurate and reproducible measurements of $\mathrm{LVH}$ are made by CMR. ${ }^{37}$ At visits 1 and 3 , CMR in the NITRATE-LVH arm and those that consent in the NITRATE-CBP arm will be used to assess LV mass, volumes and ejection fraction, T1 and T2 mapping, patterns of late gadolinium enhancement (LGE), aortic distensibility and PWV. Participants will have a peripheral venous cannula inserted and gadolinium injected at doses up to $0.2 \mathrm{mmol} / \mathrm{kg}$.

To allow a thorough assessment of cardiac function, the following imaging sequences will be undertaken:

- Scout images, including black and white blood transverse slices of the thorax.

- Long-axis cines (four-chamber, two-chamber, left ventricular outflow tract (LVOT-1 and LVOT-2), aortic valve short axis).

- Short-axis cine stack.

- T1 and T2 mapping. 
- LGE in the four-chamber, two-chamber, threechamber views and short-axis stack.

- Postcontrast T1 mapping for extracellular volume quantification.

- Sagittal aortic views and aortic flow sequences for assessment of aortic distensibility and PWV.

Short-axis images will be acquired using standard sequences, that is, 10-12 slices of $8 \mathrm{~mm}$ thickness with $2 \mathrm{~mm}$ gap to achieve whole $\mathrm{LV}$ coverage. This is undertaken with the patient in held expiration. Cine and LGE short-axis stacks will be acquired using identical scanning geometry to assure correct image registration.

The following offline parameters will be measured:

- Presence of significant extracardiac pathology.

- LV ejection fraction, volumes and mass.

- Distribution of LGE.

- Analysis of pre and post contrast T1 mapping and T2 mapping. Short-axis images will be segmented according to the American Heart Association (AHA) model as previously described. ${ }^{38}$

- Aortic distensibility and PWV.

For the analysis of study outcomes, each study will be anonymised by a third party. This will be separate from the study identification (ID) to avoid bias between pretreatment and post-treatment studies. Documentation of this anonymisation will be stored on a secure server at the Barts CVCTU, with unblinding for clinical reasons as necessary. The principal investigator (PI) will be informed if this is necessary. Study analysis will occur in batches to prevent bias. For the assessment of LV volumes and mass, two readers will undertake assessment of inter and intraobserver variation prior to analysis of blinded study images. Image analysis will be overseen and adjudicated by an experienced level three CMR reader (JCM).

\section{End of study definition}

The study will end after the final visit at week 16 .

\section{STATISTICAL ANALYSIS}

In this study, we intend to recruit 80 patients in each treatment arm with stratification of 80 patients overall recruited to NITRATE-LVH and 80 recruited to the NITRATE-CBP arms (total $\mathrm{n}=160$ ). This sample size will empower our trial to test for the primary and all major secondary endpoints listed below.

We determined the sample size using G*Power 3.0. Calculations were based on unpaired t-tests, a significance level of 0.05 (two tailed) and the relevant SD of the mean difference from published studies with reproducibility data: central systolic BP $1.7 \mathrm{~mm} \mathrm{Hg},{ }^{39} \mathrm{LV}$ mass $9.9 \mathrm{~g},{ }^{40}$ CMR PWV $0.45 \mathrm{~m} / \mathrm{s},{ }^{41}$ Vicorder PWV $0.29 \mathrm{~m} / \mathrm{s} .{ }^{41}$ For BP, we used measures of SD of systolic BP (as it has greater SD than diastolic BP) from the placebo limb of our previous intervention trial in hypertensive patients ${ }^{13}$ : clinic systolic BP $8.4 \mathrm{~mm} \mathrm{Hg}$; ambulatory systolic BP $4.9 \mathrm{~mm} \mathrm{Hg}$; home systolic BP $3.9 \mathrm{~mm} \mathrm{Hg}$. International consensus documents on the optimal method for ultrasound FMD suggest using laboratory-specific data in sample size calculation and have suggested that 40 participants in a parallel study are sufficient to determine statistically important differences. ${ }^{35}$ In a study of 4-week dietary nitrate intervention in hypertensive patients, $\mathrm{SD}$ of repeated measures of FMD in the placebo group was 0.6 , while the mean difference between the groups was $1.0 \%,{ }^{13}$ an SD of 1.1.

\section{Primary endpoints}

For the individual stratified subgroups:

\section{NITRATE-LVH}

For the primary endpoint of LV mass regression, we have a power of $95 \%$ for detecting a $9 \mathrm{~g} \mathrm{LV}$ mass change $(\sim 6 \%$ change for a mildly hypertrophied heart of $150 \mathrm{~g}$ ). Previously published data showed a $10 \%$ reduction in LV mass in hypertensives after 3 months treatment with spironolactone. ${ }^{42}$ Furthermore, dietary advice aimed at BP reduction in the Treatment of Mild Hypertension Study (TOMHS) demonstrated mean 9/9 $\mathrm{mm} \mathrm{Hg}$ BP reduction and $\sim 20 \mathrm{~g}$ LV mass reduction from baseline $\sim 200 \mathrm{~g}$ (by echocardiography) at 3 months post intervention, representing $\sim 10 \%$ reduction in LV mass. ${ }^{43}$

\section{NITRATE-CBP}

For non-CMR PWV, we have a power of $>95 \%$ for detecting a $0.6 \mathrm{~m} / \mathrm{s}$ absolute change (previously demonstrated following 4 weeks dietary nitrate in hypertensive patients ${ }^{13}$ ). For CMR determined PWV, we have a power of $>95 \%$ for detecting a $0.6 \mathrm{~m} / \mathrm{s}$ absolute change ${ }^{13}$ For our primary end point of reduction in central systolic BP, we expect a scaled reduction in BP relative to observed decreases in brachial BP $(8.7 \mathrm{~mm} \mathrm{Hg}$ clinic brachial systolic $\mathrm{BP})^{13}$ though evidence suggests that low-dose intravenous nitrite infusions act to selectively lower central BP in preference to brachial BP and therefore expected differences may be larger. ${ }^{44}$ Nevertheless, using our conservative estimate based on two-thirds brachial BP effect, we have $>90 \%$ power to detect a $5.8 \mathrm{~mm} \mathrm{Hg}$ difference in central systolic BP.

In post hoc analyses, data will be combined from the two groups to assess the overall impact of inorganic nitrate versus placebo on the entire cohort for $\mathrm{BP}$, nitrate and nitrite analysis. In addition, correlation analyses will be conducted using Pearson's correlation between measures of NOx, LV mass, BP and PWV.

\section{Secondary endpoints}

a. Ultrasound FMD (\%), >90\% power for detecting a $1.0 \%$ absolute change from a reduced FMD of $\sim 5 \% .{ }^{13}$

b. Brachial BP

i. Clinic $>85 \%$ power to detect $7 \mathrm{~mm} \mathrm{Hg}$ absolute change. ${ }^{13}$

ii. Ambulatory $>95 \%$ power to detect $7 \mathrm{~mm} \mathrm{Hg}$ absolute change. ${ }^{13}$

iii. Home $>85 \%$ power to detect $7 \mathrm{~mm} \mathrm{Hg}$ absolute change. ${ }^{13}$ The change observed in our 4 week study was $\sim 7 \mathrm{~mm} \mathrm{Hg}^{13}$ but with reduced measurement frequency, the possibility of increase measurement 
variability, and therefore, SD was arbitrarily doubled for this calculation.

Our sample size calculations assume a drop-out rate of $15 \%$ (non-compliance with dietary intervention or follow-up visits) based on our centres previous observations.

Data will be analysed on an intention-to-treat basis. The data will be compared by multivariable regression analysis for each functional measure. The change from baseline in prespecified primary, secondary and exploratory outcomes will be compared between groups by Student's t-test. We will adjust the results for multiple variables: sex, age, weight, body mass index and the initial differences between groups in LV mass.

Written approval from the research ethics committee (REC) was obtained along with final sponsorship and NHS Health Research Authority approval.

\section{Safety considerations}

The intervention is $70 \mathrm{~mL}$ of beetroot juice concentrate or nitrate-deplete beetroot juice (placebo; James White Drinks). The nitrate is extracted using the same anion exchange technique to remove inorganic nitrate from the general drinking water supplies. There are no known serious side effects from these interventions and nitratefree juice is classified as a foodstuff. In addition, several recent publications using the placebo juice are now available. ${ }^{45-47}$ In the unlikely event of a serious adverse event (SAE) occurring directly as a result of the intervention, this would need to be reviewed by the chief investigator and the procedures followed as described below.

\section{Safety reporting}

An AE will be documented in the participants' medical notes and the case report form and followed up by the investigators. SAEs will be reported to the sponsor and REC where in the opinion of the chief investigator the event was either 'related' (ie, resulted from administration of research procedures) and 'unexpected' (ie, the type of event is not an expected occurrence).

SAEs considered to be 'related' and 'unexpected' will be recorded in the participants' notes, the sponsor SAE form and reported to the sponsor's joint research management office within 24 hours of the research team being notified, and to the main REC within 15 days. The coinvestigators will be authorised to sign SAE forms in the absence of the PI. The intervention for the participant will be unblinded in the reporting of an "unexpected and related' SAE, performed by an individual independent of the study procedures and will allow the investigators to remain blinded. The unblinding of single cases will only be performed if necessary for the safety of a participant.

\section{MONITORING}

\section{Trial Steering Committee}

The trial steering committee (TSC) is composed of three independent experts in the fields of: CMR, hypertension and clinical trials along with the investigators and the data monitor and two lay members. This committee met before participant recruitment and will meet annually to assess safety, feasibility or any other arising problems and their recommendations followed.

\section{Data safety and monitoring board}

The trial is classified as low-risk and does not require the formal setting up of a data safety and monitoring board (DSMB), however, a DSMB has been established comprising a clinical trials physician, statistician and cardiovascular physician. The DSMB met to review data after 20 patients in each study arm had completed their involvement, to advise about planned sample size and safety signals. The study will be subject to monitoring by the sponsor, Queen Mary University of London, in accordance with their policies. Any monitoring findings will be relayed to the TSC by the chief investigator and acted in the best interest of patients, sponsor and funder.

\section{Ethics and dissemination}

Data collection will be completed by the third quarter of 2020. Primary and secondary analysis will start immediately after data collection is completed, with an aim to prepare publications for submission in 2021. The results of the trial will be published according to the Consolidated Standards of Reporting Trials statement. Dissemination of results will be in peer-reviewed journal publications and presentations at national and international conferences.

\section{Summary}

This is the first randomised-controlled trial assessing the use of prolonged dietary nitrate treatment in hypertension-induced target organ damage, specifically LVH and arterial stiffness. This study will determine the potential of prolonged dietary nitrate as adjunctive therapy in patients with sub optimally controlled hypertension on antihypertensive medication.

\section{Author affiliations}

${ }^{1}$ William Harvey Research Institute, Barts \& The London, Queen Mary University of London, London, UK

${ }^{2}$ Department of Cardiology, Barts Health NHS Trust, London, UK

${ }^{3} \mathrm{UCL}$ Institute of Cardiovascular Science, University College London, London, UK

Contributors All authors listed above fulfil all three International Committee of Medical Journal Editors (ICMJE) guidelines for authorship, which are (1) substantial contributions to conception and design, acquisition of data or analysis, and interpretation of data; (2) drafting the article or revising it critically for important intellectual content and (3) final approval of the version to be published. AA raised the funding. AA and VK designed the study. CWZL was responsible for coordinating the contribution of all authors to this paper. AA, VK, JCM and CWZL developed the protocol. CWZL and AJPH are involved the acquisition of data or data analysis. CWZL, KSR, JC, AS and CPP were responsible for drafting this paper. AM, CD, JCM, VK and $A A$ were responsible for editing and providing guidance on the paper. All authors were responsible for critically revising the paper. All authors approved the final version of this paper for submission. This study is supported by the CVCTU, a branch of the Barts CTU UKCRC reg no. 4.

Funding This study and CWZL are funded by The Barts Charity through a large programme grant.

Disclaimer The Sponsor and funders have no involvement in the study design, collection of data, writing of this paper or decision to submit this paper for publication. 
Competing interests $A A$ is a codirector of Heartbeet Ltd, which is a start-up company that seeks to identify commercial potential of dietary nitrate.

Patient consent for publication Not required.

Ethics approval The NIHR Barts Biomedical Research Centre Patient and Public Advisory Group reviewed this protocol prior to submission to the Research Ethics Committee (REC). The study protocol and any subsequent amendments, along with materials provided to participants and advertising material, were submitted to London - City and East REC. The study is performed in agreement with the Declaration of Helsinki and is approved by the local REC (London - City and East; 10/H0703/98).

Provenance and peer review Not commissioned; externally peer reviewed.

Open access This is an open access article distributed in accordance with the Creative Commons Attribution Non Commercial (CC BY-NC 4.0) license, which permits others to distribute, remix, adapt, build upon this work non-commercially, and license their derivative works on different terms, provided the original work is properly cited, appropriate credit is given, any changes made indicated, and the use is non-commercial. See: http://creativecommons.org/licenses/by-nc/4.0/.

ORCID iD

Clement Wai Zhen Lau http://orcid.org/0000-0003-2736-0121

\section{REFERENCES}

1 Lim SS, Vos T, Flaxman AD, et al. A comparative risk assessment of burden of disease and injury attributable to 67 risk factors and risk factor clusters in 21 regions, 1990-2010: a systematic analysis for the global burden of disease study 2010. Lancet 2012;380:2224-60.

2 Forouzanfar MH, Liu P, Roth GA, et al. Global burden of hypertension and systolic blood pressure of at least 110 to $115 \mathrm{MM} \mathrm{Hg}, 1990-$ 2015. JAMA 2017;317.

3 Vos T, Alemu Abajobir A, Hassen Abate K, et al. Global, regional, and national incidence, prevalence, and years lived with disability for 328 diseases and injuries for 195 countries, 1990-2016: a systematic analysis for the global burden of disease study 2016. Lancet 2017;390:1211-59.

4 WHO. Global status report on noncommunicable diseases 2014. Geneva, 2014. Available: http://apps.who.int/iris/bitstream/handle/ 10665/148114/9789241564854_eng.pdf;jsessionid=FD9481BC2C21 3324152C9CDB8D3740DF? sequence $=1$

5 Appel LJ, Moore TJ, Obarzanek E, et al. A clinical trial of the effects of dietary patterns on blood pressure. N Engl J Med 1997;336:1117-24.

6 Joshipura KJ, Hu FB, Manson JE, et al. The effect of fruit and vegetable intake on risk for coronary heart disease. Ann Intern Med 2001;134:1106.

7 Lundberg JO, Feelisch M, Björne $\mathrm{H}$, et al. Cardioprotective effects of vegetables: is nitrate the answer? Nitric Oxide 2006;15:359-62.

8 Ghosh SM, Kapil V, Fuentes-Calvo I, et al. Enhanced vasodilator activity of nitrite in HypertensionNovelty and significance. Hypertension 2013;61.

9 Kapil V, Milsom AB, Okorie M, et al. Inorganic nitrate supplementation lowers blood pressure in humans. Hypertension 2010;56:274-81.

10 Webb AJ, Patel N, Loukogeorgakis S, et al. Acute blood pressure lowering, vasoprotective, and antiplatelet properties of dietary nitrate via bioconversion to nitrite. Hypertension 2008;51:784-90.

11 Tannenbaum SR, Weisman M, Fett D. The effect of nitrate intake on nitrite formation in human saliva. Food Cosmet Toxicol 1976;14:549-52.

12 Duncan $\mathrm{C}$, Dougall $\mathrm{H}$, Johnston $\mathrm{P}$, et al. Chemical generation of nitric oxide in the mouth from the enterosalivary circulation of dietary nitrate. Nat Med 1995;1:546-51.

13 Kapil V, Khambata RS, Robertson A, et al. Dietary nitrate provides sustained blood pressure lowering in hypertensive patients: a randomized, phase 2, double-blind, placebo-controlled study. Hypertension 2015;65:320-7.

14 Greenwald SE. Ageing of the conduit arteries. J Pathol 2007:211:157-72.

15 Laurent S, Cockcroft J, Van Bortel L, et al. Expert consensus document on arterial stiffness: methodological issues and clinical applications. Eur Heart J 2006;27:2588-605.

16 Ben-Shlomo Y, Spears M, Boustred C, et al. Aortic pulse wave velocity improves cardiovascular event prediction. J Am Coll Cardiol 2014;63:636-46.

17 Boutouyrie P, Laurent S, Briet M. Importance of arterial stiffness as cardiovascular risk factor for future development of new type of drugs. Fundam Clin Pharmacol 2008;22:241-6.
18 Gradman AH, Alfayoumi F. From left ventricular hypertrophy to congestive heart failure: management of hypertensive heart disease. Prog Cardiovasc Dis 2006;48:326-41.

19 Nadruz W. Myocardial remodeling in hypertension. J Hum Hypertens 2015;29:1-6.

20 Levy D, Garrison RJ, Savage DD, et al. Prognostic implications of echocardiographically determined left ventricular mass in the Framingham heart study. N Engl J Med 1990;322:1561-6.

21 Kannel WB. Left ventricular hypertrophy as a risk factor in arterial hypertension. Eur Heart J 1992;13:82-8.

22 Ruilope LM, Schmieder RE. Left ventricular hypertrophy and clinical outcomes in hypertensive patients. Am J Hypertens 2008;21:500-8.

23 Mathew J, Sleight P, Lonn E, et al. Reduction of cardiovascular risk by regression of electrocardiographic markers of left ventricular hypertrophy by the angiotensin-converting enzyme inhibitor ramipril. Circulation 2001;104:1615-21.

24 Dahlöf B, Devereux RB, Kjeldsen SE, et al. Cardiovascular morbidity and mortality in the losartan intervention for endpoint reduction in hypertension study (life): a randomised trial against atenolol. The Lancet 2002;359:995-1003.

25 Ruiz-Hurtado G, Delgado C. Nitric oxide pathway in hypertrophied heart: new therapeutic uses of nitric oxide donors. J Hypertens 2010;28:S56-61.

26 Carlström M, Persson AEG, Larsson E, et al. Dietary nitrate attenuates oxidative stress, prevents cardiac and renal injuries, and reduces blood pressure in salt-induced hypertension. Cardiovasc Res 2011;89:574-85.

27 Bahra M, Kapil V, Pearl V, et al. Inorganic nitrate ingestion improves vascular compliance but does not alter flow-mediated dilatation in healthy volunteers. Nitric Oxide 2012;26:197-202.

28 Ikonomidis I, Aboyans V, Blacher J, et al. The role of ventriculararterial coupling in cardiac disease and heart failure: assessment, clinical implications and therapeutic interventions. A consensus document of the European Society of Cardiology Working Group on Aorta \& Peripheral Vascular Diseases, European Association of Cardiovascular Imaging, and Heart Failure Association. Eur $\mathrm{J}$ Heart Fail 2019;21:402-24.

29 Lang RM, Badano LP, Mor-Avi V, et al. Recommendations for cardiac chamber quantification by echocardiography in adults: an update from the American Society of echocardiography and the European association of cardiovascular imaging. Eur Heart $J$ Cardiovasc Imaging 2015;28:1-39.

30 Devereux RB, Alonso DR, Lutas EM, et al. Echocardiographic assessment of left ventricular hypertrophy: comparison to necropsy findings. Am J Cardiol 1986;57:450-8.

31 Ignarro LJ, Fukuto JM, Griscavage JM, et al. Oxidation of nitric oxide in aqueous solution to nitrite but not nitrate: comparison with enzymatically formed nitric oxide from L-arginine. Proc Natl Acad Sci U S A 1993;90:8103-7.

32 Bush PA, Gonzalez NE, Griscavage JM, et al. Nitric oxide synthase from cerebellum catalyzes the formation of equimolar quantities of nitric oxide and citrulline from L-arginine. Biochem Biophys Res Commun 1992;185:960-6.

33 Hickson SS, Butlin M, Broad J, et al. Validity and repeatability of the Vicorder apparatus: a comparison with the SphygmoCor device. Hypertens Res 2009;32:1079-85.

34 Celermajer DS, Sorensen KE, Gooch VM, et al. Non-Invasive detection of endothelial dysfunction in children and adults at risk of atherosclerosis. The Lancet 1992;340:1111-5.

35 Corretti MC, Anderson TJ, Benjamin EJ, et al. Guidelines for the ultrasound assessment of endothelial-dependent flowmediated vasodilation of the brachial artery: a report of the International brachial artery reactivity Task force. J Am Coll Cardiol 2002;39:257-65.

36 Wharton G, Steeds R, Allen J, et al. A minimum dataset for a standard adult transthoracic echocardiogram: a guideline protocol from the British Society of echocardiography. Echo Res Pract 2015;2:G9-24.

37 Bottini P, Carr A, Prisant L, et al. Magnetic resonance imaging compared to echocardiography to assess left ventricular mass in the hypertensive patient. Am J Hypertens 1995;8:221-8.

38 Treibel TA, Kozor R, Schofield R, et al. Reverse Myocardial Remodeling Following Valve Replacement in Patients With Aortic Stenosis. J Am Coll Cardiol 2018;71:860-71.

39 Siebenhofer A, Kemp C, Sutton A, et al. The reproducibility of central aortic blood pressure measurements in healthy subjects using applanation tonometry and sphygmocardiography. J Hum Hypertens 1999;13:625-9.

40 Hudsmith LE, Petersen SE, Francis JM, et al. Normal human left and right ventricular and left atrial dimensions using steady state free 
precession magnetic resonance imaging. J Cardiovasc Magn Reson 2005;7:775-82.

41 Grotenhuis HB, Westenberg JJM, Steendijk P, et al. Validation and reproducibility of aortic pulse wave velocity as assessed with velocity-encoded MRI. J. Magn. Reson. Imaging 2009;30:521-6.

42 Gaddam K, Corros C, Pimenta E, et al. Rapid reversal of left ventricular hypertrophy and intracardiac volume overload in patients with resistant hypertension and hyperaldosteronism. Hypertension 2010;55:1137-42.

43 Liebson PR, Grandits GA, Dianzumba S, et al. Comparison of five antihypertensive monotherapies and placebo for change in left ventricular mass in patients receiving nutritional-hygienic therapy in the treatment of mild hypertension study (TOMHS). Circulation 1995;91:698-706 http://www.ncbi.nlm.nih.gov/pubmed/7828296
44 Omar SA, Fok H, Tilgner KD, et al. Paradoxical Normoxia-Dependent selective actions of inorganic nitrite in human muscular conduit arteries and related selective actions on central blood pressures. Circulation 2015:131:381-9.

45 Gilchrist M, Winyard PG, Fulford J, et al. Dietary nitrate supplementation improves reaction time in type 2 diabetes: development and application of a novel nitrate-depleted beetroot juice placebo. Nitric Oxide 2014;40:67-74.

46 Gilchrist M, Winyard PG, Aizawa K, et al. Effect of dietary nitrate on blood pressure, endothelial function, and insulin sensitivity in type 2 diabetes. Free Radic Biol Med 2013;60:89-97.

47 Lansley KE, Winyard PG, Bailey SJ, et al. Acute dietary nitrate supplementation improves cycling time trial performance. Med Sci Sports Exerc 2011;43:1125-31. 